\title{
CAZADORES DE HUELLAS INDICIALES DEL ESTAR SIENDO (ABIERTO). EL CASO DE LAS ECONOMÍAS AMALGAMADAS DE KARL POLANYI
}

\author{
Pedro Huerta ${ }^{1}$
}

\section{Resumen/Abstract}

El presente ensayo, constituye una aproximación de lectura a un autor especifico, el cual presenta un conjunto de paralelismo con otros cazadores indiciarios (autores) que pensaron la "sociedad" en la perspectiva de los fragmentos, los detalles infinitesimales y las asociaciones. Es un estudio de caso/autor del cual se desprenden una serie de dimensiones a explorar en conexión con pensadores como Simmel, Elias, Tarde, Certeau y Deleuze. En definitiva una tentativa de lectura a cazadores de huellas indíciales de un proyecto mayor, que se conecta con el desarrollo de un utillaje mental ${ }^{2}$ para pensar el desarrollo de la transdisciplinariedad en ciencias sociales.

Palabras clave: transdisciplinariedad, artes de hacer, huellas indiciarias, economía sustantiva, dispositivos socioculturales, cazadores indiciarios.

\section{HUNTERS OF INDIRECT FOOTPRINTS OF BEING (OPEN). THE CASE OF KARL POLANYI'S AMALGAMATED ECONOMIES}

The present essay constitutes a reading approach to a specific author, which presents a set of parallelism with other indicial hunters (authors) who thought about "society" in the perspective of fragments, infinitesimal details and associations. It is a case study / author from which emerge a series of dimensions to be explored in connection with thinkers such as Simmel, Elias, Tarde, Certeau and Deleuze. In short, an attempt to read to hunters of indigenous traces of a larger project, which is connected with the development of a mental tool to think about the development of transdisciplinary in social sciences.

Keywords: transdisciplinarity, arts of doing, fingerprinting, substantive economy, socio-cultural devices, fingerprinting hunte.

Dios está en lo particular (Aby Warburg)

El cazador habría sido el primero en "contar una fábula” porque era el único en condiciones de leer, en las huellas mudas (sino imperceptibles) dejadas por la presa, una serie coherentes de acontecimientos (Carlo Ginzburg, 2004)

\footnotetext{
${ }^{1}$ Chileno, Universidad Academia de Humanismo Cristiano. E-mail: phuerta@academia.cl

${ }^{2}$ En Historia de las Mentalidades se utiliza el termino de utillaje mental para referirse a un vocabulario, lugares comunes, cuadros lógicos, etc.
} 


\section{Introducción}

Durante milenios el hombre fue cazador. En el curso de las persecuciones

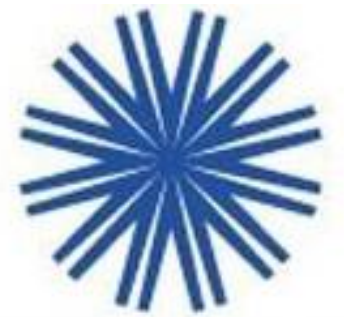

innumerables aprendió a reconstruir las formas y los movimientos de las presas invisibles partiendo de huellas en el fango, ramas rotas, bolas de estiércol, mechones de pelos, plumas enredadas, olores estancados. Aprendió a husmear, clasificar huellas infinitesimales como hilos de baba. Aprendió a realizar omplejas con rapidez fulmínea, en la espera del bosque o en un claro lleno de traicioneras amenazas (Ginzburg, 2004). En las páginas que siguen intentaré mostrar cómo el economista Karl Polanyi se ha transformado en un cazador de aspectos marginales, a veces pasados por alto en la teoría económica convencional. La perspectiva de Polanyi posee cierto grado de concordancia con las prácticas del acontecer contemporáneo de lo que Bruno Latour ha llamado "la sociología de las asociaciones", de los ensamblajes, las interconexiones, los entrecruces, en el marco de la pluralidad de los modos del estar siendo en la relación con otros. Adquiere también sintonía con una serie de autores que hemos denominado metafóricamente como cazadores de huellas indiciarias del fenómeno del estar siendo de la socialidad, pero también en este caso de estudio de las prácticas que llamamos económicas, en tanto campo de la producción, la distribución y el consumo.

Si bien vamos a partir con el estudio de caso de un autor especifico que es Karl Polanyi, dejaremos planteado en la parte final de este trabajo, que lo anterior constituye un punto de partida en la exploración de autores que presentan una serie de paralelismos inscritos en el plano de la "socialidad", en su modo relacional e interdependiente, que es preciso abarcar en sus rendimientos para el estudio de ésta y de las practicas económicas, y de su conexión más amplia con la política en su sentido crítico-emancipatorio.

La metáfora del cazador de las huellas indiciarias intenta mostrar a Karl Polanyi como un cazador de lo imperceptible, de lo no visto, de lo pasado por alto, o considerado muchas veces como trivial o poco relevante para las economías dominantes, cuestión que permite expandir la mirada a nuevas maneras de entender aspectos antes no significados y que se tornan fundamentales para la compresión de los fenómenos de las economías contemporáneas y de las sociologías de las asociaciones o ensamblajes (Latour, 2008). Esto abre también la posibilidad de pensar lo "social" en una serie de autores que presentan 
similitud con los planteamientos de Polanyi, pero desde la dimensión de la socialidad o del socius (Simmel, Elias, Tarde, De Certeau, Deleuze).

He tomado los adjetivos de lo abierto y lo cerrado del crítico literario brasileño Santiago Silviano, desarrollado en su artículo "Interpretando interpretaciones de América Latina" (Santiago 2012). En principio, lo que quiero destacar de Santiago Silviano es su lectura del "entre" o al medio "de" disciplinas que se entrecruzan y que encuentran salida en una hermenéutica posestructuralista del laberinto de la soledad del escritor mexicano Octavio Paz. Los adjetivos de lo abierto y lo cerrado en Octavio Paz, en la lectura de Silviano, se encuentran a lo menos en dos cadenas de significación. La primera es la que abre el tema de género, que se sustenta en la visión del autor sobre el hombre y la mujer mexicana, y la rebaja de la última a la condición de chingada (un ser abierto), valorando al primero en su condición de patriarca (ser cerrado). La segunda cadena de significación donde aparece los adjetivos de abierto y cerrado se encuentra en los proyectos liberales y católicos en América Latina que representan lo cerrado. Lo abierto seria lo que no puede ser clausurado en una definición univoca, simétrica y homogénea, una especie de entre o para el caso de nuestro planteamiento una anomalía, un elemento inclasificable o aun no clasificado en la teoría convencional de las prácticas "económico-sociales”.

\section{Un caso de estudio.}

Las economías como un estar siendo (abierto) meta-utilitarista en Karl Polanyi ${ }^{3}$

Lo "social" en un sentido abierto no universal, también hace alusión a lo económico ${ }^{4}$, como también a otras esferas de las socialidades, un concepto de realidad total en su proceso de acontecer, relacional e interdependiente. Por necesidad o comodidad técnica los cientistas sociales terminaron parcelando en la búsqueda de lo que Max Weber denominó tipos ideales, simplificación de realidad para construir categorías a propósito de cuestiones significativas.

\footnotetext{
${ }^{3}$ El concepto de metautilitarismo de la economía social y solidaria lo utiliza el teórico brasileño Luiz Inacio Gaiger "para desmentir la tesis de la racionalidad única o de la existencia de un modelo intrínsecamente superior. Sus prácticas atestan la multiplicidad, las constantes composiciones entre fundamentos y modos de actuar, indisociables de las cosas y de las animas, como decía Mauss." (Gaiger 2017).

${ }^{4}$ A partir de Polanyi entendemos lo económico como el proceso/acontecer/movimiento institucionalizado de interacción entre el hombre y el medio, dándole a esta interacción, de manera continuada, los medios materiales de satisfacer sus necesidades. (Polanyi 1983).
} 
Esto ha sido parte de la especialización disciplinaria y la modernización de las ciencias sociales en general, que a partir de la década del 50 del siglo XX fueron interpeladas por esa necesidad política denominada “desarrollo”. En ese marco intelectual la economía convencional fue cada vez más asimilada por un tipo de economía que Polanyi denominó economía formal.

Los supuestos que están a la base de estas teorizaciones de economía formal, son la búsqueda de la riqueza como norte de todas las prácticas económicas, lo que consolida la elaboración de un concepto de economía que da cuenta de las necesidades lucrativas y las maneras de impulsar esta finalidad. A partir de las premisas anteriores, aparece el análisis formal de la economía. El homo oeconomicus constató la insuficiencia de medios (la escasez) y actuó racionalmente maximizando, economizando y aumentando los medios para satisfacer esos fines ilimitados. Este enunciado fundó dos premisas básicas en la economía moderna el ahorro y la productividad. La acción económica moderna en este enfoque convencional, en la lógica de la acción racional, fue aplicada a todos los medios y fines que abarca la casi infinita variedad de intereses humanos.

Bajo los supuestos de este enfoque, el hombre económico actúa per se, maximizando y racionalizando, ya que se encuentra sometido a la escasez. Ello otorga a las prácticas económicas un carácter generalizable y estático, dado que en todas las circunstancias el homus oeconomicus actuaría en consonancia al principio de la maximización, buscando la mayor rentabilidad dentro de sus posibilidades. En el marco de estos supuestos ¿es posible comprender a las comunidades pastoriles de Cerdeña y a muchas otras? Ellas organizaban periódicamente fiestas colectivas en las que despilfarraban gran parte de su excedente, a expensas de su capacidad de ahorrar e invertir, vale decir de su acción maximizadora.

Si nos guiamos por el modelo convencional formal de la economía, donde el hombre en todas sus circunstancias actúa maximizando y racionalizando, dicho comportamiento económico sería aplicable a todas las expresiones del devenir social. Estas premisas establecen un análisis universal-estático de las prácticas económicas, a partir de un método deductivo y general.

En contraposición, Polanyi planteo un punto de vista diferente de lo "económico" o las "economías", que parte inductivamente de las prácticas económicas, donde lo económico es posible en su desenvolvimiento contingente amalgamado o incrustado en las dimensiones de la vida social de las interacciones e 
interdependencias, cuestión irrelevante para la teoría convencional de la economía. De Polanyi se desprende una crítica a la teoría económica convencional univoca. A partir de las categorizaciones abstractas y universales, desprovistas de comprobación en las prácticas efectivas, se generalizó un cierto universal de economía en la sociedad - de la dinámica del acontecer de la economía-, en la perspectiva de lo simétrico y lo universal, con categorías cerradas o estancadas,

En este sentido, se evidencia una crítica a posiciones que el autor denomina empiristas-abstractas, (cerradas) construidas, sobre la base de categorías de análisis vacías, en términos de su contenido práctico (acontecer) y antropológico (cultural). En este plano su principal crítica es a la obra de Talcott Parsons que suponía al mercado como la institución económica prototípica y a la lógica de la acción racional de los intercambios mercantiles como la base de una teoría universal de las acciones humanas. (Lo universal=lo cerrado).

Para los efectos de ampliar los márgenes analíticos de la economía, en la lógica de la pluralidad y las practicas, Polanyi, propone una definición que le permitiese abarcar una variedad de formas posibles de economía (una visión plural), partiendo de la idea de que no existe "la economía”, desprovista de cualquier prejuicio, en cuanto a cuáles formas eran posibles y cuáles no, ya que lo importante es remitirse a su comportamiento practico, es decir, a su funcionamiento en el acontecer de lo que llamamos economía.

A tal definición de las economías en su sentido plural la denominó como real o substantiva, una visión de la economía entendida, no solo como pretensión de economización racional universal a priori, sino como las múltiples formas que adquiere la búsqueda del sustento del hombre en sociedad, cuyo funcionamiento es posibilitado por los dispositivos culturales y sociales, desde donde se instituye lo económico en el acontecer de las prácticas sociales.

En esta perspectiva, el estudio de la economía, debía ser comprendido en las prácticas específicas que adquiere el estar siendo de lo económico, en las interdependencias sociales, y en perspectiva de su estructuración (historia). Estas acciones o prácticas relacionales efectivas y cotidianas expresan la substancia de las categorías elaboradas en el modo de análisis polanyiano de la economía como acontecer más allá del utilitarismo. 
El despliegue de la economía como acontecer/proceso/movimiento.

El punto de vista analítico de Karl Polanyi se sustenta en la definición real o sustantiva de la economía, que surge del proceso de institucionalización de la interacción e interdependencias entre el hombre y el medio natural y social, que dota a estas interacciones de manera continuada de los medios materiales para satisfacer sus necesidades (Polanyi 1976: 159). A partir de esta definición, Polanyi elabora un punto de vista analítico que entiende la economía como proceso/acontecer y movimiento, en el cual son instituidas las prácticas que llamamos económicas. La institucionalización de estos procesos/practicas económicas se manifiesta en los movimientos de transacción y disposición en el modo en que estas prácticas se instituyen en la socialidad.

Respecto del concepto de "proceso", que puede ser leído como acontecer/movimiento de prácticas económicas que se dan en "cambios de localización o bien cambios de apropiación o bien ambos” (Polanyi 1976:159), expresadas en el acontecer de las practicas económicas, se pueden manifestar a lo menos en dos tipos de movimientos/acontecimiento. Por una parte, en el de localización que incluye la producción y el transporte para "el cual el traslado espacial del objeto es igualmente esencial” (Ibíd.). A su vez, el movimiento de apropiación "designa generalmente la circulación de bienes como su administración” (Ibíd.). En el primer caso, el movimiento de apropiación es el resultado de una transacción; en el segundo de disposición socio-cultural para el hacer.

Por transacción, Polanyi entiende "un movimiento de apropiación que ocurre entre manos" (Ibíd.), el termino mano se utiliza en el autor para denominar los cargos y los organismos públicos, así como las personas o firmas privadas, siendo la diferencia entre ellas una cuestión de organización interna. Por disposición, entiende las fuerzas de la costumbre o de la ley a las cuales se le atribuye efectos concretos de apropiación.

(...) Las actividades sociales en la medida que forman parte del proceso, pueden denominarse económicas; las instituciones se denominan así en la medida en que contienen una concentración de tales actividades: todos los componentes que forman parte del proceso pueden considerarse elementos económicos. (Polanyi 1976:160).

La idea anterior es decidora en dos planos, primero, fundar un punto de vista de análisis económico más allá del mero reduccionismo economicista, y segundo, el rol que juegan las relaciones e interacciones 
socioculturales en el acontecer de las practicas económicas, entendidas como construcciones y disposiciones sociales donde lo económico adquiere valides y consistencia. Éstas se manifiestan en disposiciones culturales y socialidades que dan unidad y estabilidad al proceso económico en el tiempo y espacio, es decir, en la manera que los procesos entendidos como movimientos económicos de “apropiación” y “disposición” son instituidos. El modo de institucionalización del proceso económico, entonces, se produce a partir de dos categorías que el autor expone con bastante claridad en el siguiente párrafo:

(...) La institucionalización del proceso económico dota al proceso de unidad y estabilidad crea una estructura con una función determinada en la sociedad; traslada el lugar del proceso en la sociedad añadiendo de este modo significación a su historia; centra el interés en los valores, los motivos y la política. Unidad y estabilidad, estructura y función, historia y política deletrean de forma operacional el contenido de nuestra afirmación de que el sistema económico humano es un proceso institucionalizado. (Polanyi 1976:161).

Siguiendo el punto de vista polanyiano, la puesta en práctica de la institucionalización de las actividades económicas, entendidas como procesos de movimiento de transacción y disposición, se generan a partir de la unidad, en tanto estructuración de estos procesos en el tiempo (historia) y estabilidad, como la función de estas prácticas en la contingencia. La política sería el momento de estabilidad o de cierre de las practicas económicas, también denominada modo de hacer, cuya función es la de motor o de engranaje, donde no se descarta la innovación y el cambio, que es también expresión del equilibrio de las fuerzas en lucha en las socialidades. A partir de ello, se desprende que para Polanyi las prácticas económicas de los individuos están incrustadas y amalgamadas en instituciones culturales, en lo modos de hacer. En el arte de hacer esto o aquello, para ponerlo en la perspectiva de Michel de Certau (1996).

El punto analítico de Polanyi, nos permite ir más allá de una simple apendización de lo social, lo político y lo cultural en el proceso económico, en tanto lo económico es un hecho intersticial de las artes de hacer (Certeau) en sus movimientos contingentes que terminan instituyendo los modos de hacer de la economía en las culturas y la socialidad, al decir de Polanyi:

(...) La economía humana, pues, esta incrustada y enredada en instituciones económicas y no económicas. La inclusión de lo no económico es vital. Pues la religión o el gobierno pueden ser tan importantes para la estructura y el funcionamiento de la economía como las instituciones monetarias o la 
disponibilidad de herramientas y maquinas que aligeran el trabajo de la mano de obra. (Polanyi 1976:161).

Las Artes de hacer (Certeau). Reciprocidad, redistribución e intercambio.

La perspectiva de la economía como acontecer y movimiento se funda en el estudio de las practicas económicas como un estar siendo no estático, es decir, como movimientos que dan paso a las diversas formas en las cuales se instituye lo económico en las socialidades y modos de hacer a lo largo del tiempo. Polanyi establece que la configuración de economías empíricas se produce por medio de la interdependencia y recurrencia, es decir, en la combinación de "formas de integración", las que se presentan coetáneamente, en diferentes niveles y sectores de la economía que, a lo largo del devenir de la historia humana, se manifiestan sin imposición de una forma de integración por sobre otras.

Empíricamente encontramos que las principales pautas son la reciprocidad, la redistribución y el intercambio. La reciprocidad denota movimientos entre puntos correlativos de agrupamiento simétricos; la redistribución designa movimientos de apropiación hacia un centro y luego hacia el exterior; el intercambio hace referencia aquí a movimientos y viceversa en un sentido y en el contrario que tiene lugar como entre manos en el sistema de mercado. (Polanyi 1976:162).

Siguiendo el esquema analítico de Polanyi, las formas de integración no son agregados de comportamiento interpersonales ni individuales, más bien, sus posibilidades dependen de determinados dispositivos instituyentes como las organizaciones asimétricas centralizadas que operan en las prácticas de redistribución, o los sistemas de organización simétricos que posibilitan las prácticas de reciprocidad. De manera que las posibilidades de desarrollo de estas formas de hacer dependen, en esencia, de apoyos institucionales concretos:

(...) En la esfera económica es tan frecuente que el comportamiento interpersonal no tenga los esperados efectos en ausencia de unas concretas precondiciones institucionalizadas. Solo en un medio ambiente simétricamente organizado producirá el comportamiento reciproco instituciones económicas de cierta importancia; solo donde se ha creado centros distribuidores pueden producir los actos individuales de compartir una economía redistributiva; y solo en presencia de un sistema de formación de precios por el mercado tendrán como consecuencia los actos individuales de intercambio unos precios fluctuantes que integren la economía. En otros casos tales trueques resultarán ineficaces y, por tanto, tenderán a no producirse. Caso de que, no obstante, ocurra, de forma azarosa, se despertara una violenta reacción emocional, como contra los 
actos indecentes o los actos de traición, puesto que el comportamiento comercial no es un comportamiento emocionalmente indiferente y, por lo tanto, la opinión no lo tolera fuera de los canales aprobados" (Polanyi 1976:163).

Reciprocidad, redistribución e intercambio son formas modélicas de las artes del hacer en las que se expresa lo instituyente de la economía en la sociedad, ya que estas operaciones económicas suceden y están sucediendo en la interdependencia y la recurrencia de los movimientos económicos en los modos de sociabilidad. Estas formas, se instituyen a partir de los conceptos de unidad y estabilidad, es decir, estructuras de prácticas económicas que se expresan a lo largo del tiempo (unidad) y acciones recurrentes exitosas que decantan en la estabilidad del sistema (estabilidad), que, como lo menciona Polanyi, contienen las expresiones de violencia emocional. La institucionalización de estas formas de integración económica, que se pueden dar coetáneamente, se expresan en una doble dimensión analítica, una de carácter histórico, que se desarrolla en el modo como se estructuran las actividades económicas en el largo plazo, y otra de carácter político que se manifiesta en la función contingente de determinadas prácticas económicas en las interacciones sociales, como acciones que posibilitan la estabilidad de un cierto orden social.

La reciprocidad como forma de integración o como arte de hacer, supone relaciones sociales simétricas entre individuos, de manera que para su institucionalización económica necesita de prácticas, operaciones o modos de empleo facilitadores de organización simétrica, cuyo ejemplo más conocido son los sistemas simétricos de parentesco o filiación. Cabe señalar, que estas artes del hacer lo económico se dan en diferentes niveles y sectores de la actividad económica, además están amalgamadas con las otras formas de integración. En una unidad económica de tipo doméstica o familiar, por ejemplo, se pueden dar formas de reciprocidad y redistribución paralelamente, lo que las diferenciará serán las pautas institucionales facilitadoras de las mismas que le darán la esencia a su operatoria. Para el caso de la redistribución, la asimetría, y para el caso de la reciprocidad la simetría. Sin estas operaciones o modos de empleo facilitadores, estas prácticas no se podrían anclar en el proceso económico concreto.

La reciprocidad como forma de integración gana fuerza en su capacidad de utilizar tanto la redistribución como intercambio a manera de métodos subordinados. La reciprocidad puede conseguirse gracias a compartir una carga de trabajo de acuerdo a determinadas formas de redistribución, como cuando se cogen las cosas por turno. De manera similar, la reciprocidad se consigue a veces mediante intercambios de determinadas equivalencias en beneficio del asociado que esta escaso de alguna clase de necesidades (una 
institución fundamental de las sociedades orientales antiguas). En las economías sin mercado estas dos formas de integración-la reciprocidad y la redistribución-suelen de hecho presentarse juntas" (Polanyi 1976:164).

Por otra parte, la redistribución se puede expresar de la siguiente manera:

"La redistribución se consigue dentro de un grupo, en la medida en que la asignación de los bienes se reúna en una mano y tenga lugar en virtud de la costumbre, la ley o una decisión central ad hoc. A veces esto significa una reunión física, acompañada de almacenamiento y redistribución, y otras veces la reunión no es física, sino simplemente apropiativa, es decir, el derecho a disponer de la localización física de los bienes. La redistribución se presenta por muchas razones, en todos los niveles de la civilización, desde la tribu cazadora primitiva hasta los grandes sistemas de almacenamiento de Egipto, Sumeria, Babilonia y Perú. En los grandes países, la diferencia de tierra y clima pueden hacer necesaria la redistribución; en otros casos se debe la relevancia a lo relativo al tiempo como ocurre entre la recolección y el consumo. En el caso de la caza, cualquier otro método de distribución conduce a la desintegración de la horda o banda, puesto que en este caso solo la "división del trabajo" puede asegurar los resultados; la redistribución del poder de compra puede ser valiosa en sí misma, es decir, para los propósitos que exigen los ideales sociales de los modernos estados de bienestar. El principio sigue siendo el mismo: reunir y redistribuir desde un centro" (Polanyi 1976:165).

Cabe señalar que la redistribución no es una cualidad específica de grandes unidades económicas, tanto comunitarias como societales; también ésta puede darse en unidades de menor tamaño como las unidades domésticas o familiares. Al respecto, Polanyi cita los ejemplos de la "Kraal de África central, la familia patriarcal hebrea, la hacienda griega de la época de Aristóteles, la familia romana, el feudo medieval o la típica gran familia campesina anterior a la comercialización general de los cereales. El principio sigue siendo el mismo: reunir y redistribuir desde un centro" (Polanyi 1976:165).

Con respecto al intercambio, Polanyi va más allá de una perspectiva que asocie intercambio a mercado autoregulado, en otras palabras, el intercambio. Si bien, se da esencialmente en el ámbito del mercado, este no es una institución genérica y adquiere especificidades en sus contextos de realización; es también, en la lógica de este cazador de lo indiciario, una construcción cultural. Este economista plantea que el intercambio de mercado formadores de precios, es uno entre otras formas de intercambio, en tanto se puede dar intercambios a precios fijos en mercados administrados, donde el intercambio no revestiría formaciones de precios. Al respecto, Polanyi distingue tres maneras que adquiere el intercambio en las sociedades: 
El movimiento meramente locacional de cambio de lugar entre manos (intercambio operacional); los movimientos aproximativos de intercambio con un índice fijo (intercambio decisional) o con un índice contractual (intercambio integrador) En la medida que se trata del intercambio fijo, el sistema está integrado por factores que fijan ese índice, no mediante el mecanismo de mercado. Incluso los mercados formadores de precios solo son integradores si están vinculados en un sistema que tiende a extender el efecto de los precios a otros mercados distintos de los directamente afectados" (Polanyi, 1976:165).

Estos tres artes de hacer, en el sentido de los modos de empleo de operatorias económicas desarrolladas en las practicas, que se han ido instituyendo en los principios de la reciprocidad, redistribución e intercambio, no constituyen etapas del desarrollo económico y social, como tampoco implican un orden temporal progresivo, como se suele interpretarse superficialmente. Estas formas o modos de hacer la economía, pueden manifestarse paralelamente y en diferentes estadios temporales, en tanto, unas pueden ser más preponderantes en determinados periodos que otras. Estos modos de hacer de la economía no constituyen, a su vez, un análisis de tendencia estructural, ni un modelo de etapas de desarrollo; más bien, son formas singulares en las que se instituye las economías en su sentido plural en las artes del hacer, las cuales han presentado cierta recurrencia e interdependencia en el acontecer de las practicas económicas a través de su estabilidad en el tiempo. Por tanto, no deben ser entendidas como categorías estáticas, tampoco como tendencias generales universalizables, sino como modos de integración, artes de hacer, operatorias, procedimientos, maneras de hacer de las economías en tanto dinámicas socio-culturales

El modo de abordar la economía en Polanyi es un modelo opuesto al materialismo histórico, concebido como etapas de desarrollo insostenible en términos históricos. El modo polanyiano es un tipo de análisis económico inductivo, empirista, teórico y crítico, sustentado en la historia y la antropología económica, del cual se desprende un análisis de la pluralidad económica cuyos rendimientos son sustanciales para pensar los espacios intersticiales de los entres y de las nuevas expresiones de la economía que apuntan en buena medida "a defender o explorar la idea de que el fomento de las prácticas y unidades económicas de tipo cooperativo, asociativo, comunitario, solidario, debiesen ser comprendidos como el corazón central en la constitución de una "economía social” (Gonzalez 2016:7) y de una sociología de los ensamblajes (Latour,2008). 


\section{Una variante a explorar. Algunos cazadores indiciarios de las socialidades.}

Existe una serie de autores (cazadores indiciarios) que pensaron en la operaciones multiformes y fragmentarias de lo "social" y que al igual que Polanyi se contraponían a la idea de las grandes interpretaciones de lo social, la sociedad o la economía y que se inscribe en la tradición que Bruno Latour (2008) ha denominado como "sociología de las asociaciones". En contraposición a ello expusieron lecturas sobre lo social en su sentido abierto, desde la perspectiva de los pequeños fragmentos, el rescate de la singularidad en oposición a las generalizaciones, una sociología muchas veces outsider del sistema universitario, a veces olvidada, donde se incluyó al individuo en tanto interacción, el rechazo a la explicación basada en los grandes hombres "remplazándolas por las pequeñas ideas de los pequeños hombres, pequeñas invenciones" (Tarde 2011). Son los planteamientos de algunos de estos cazadores de lo indiciario que rescato a modo de cierre del presente ensayo y que encuentran sintonía con los planteamientos del autor antes analizado (Karl Polanyi). Un primer autor a relevar en este sentido es Norbert Elias (1994) quien, de cierto modo, es un intelectual global, por sus contribuciones a lo que podríamos llamar el campo de lo "social", lo "histórico", pero también lo "cultural" y lo "político" desde la perspectiva de sus estudios "psicogéneticos y sociogéneticos", donde se correlacionan estructura psíquica, estructura social y poder. Para Elias el proceso de la civilización es el resultado de redes de relaciones que se producen en la correlación de los cambios estructurales (centralización del poder político), los cambios en la estructura social (cambio en los modos de convivencia) y los cambios en la estructura psíquica (coacciones y autocontrol de los impulsos que operan en el plano de las emociones como el pudor o el asco).

Para Elias el proceso de la civilización, si bien no es un proceso determinado por ninguna prescripción o planificación racional, sigue un cierto orden dado por el entrecruce de las dimensiones del poder, la convivencia y los individuos en sus interdependencias. Para Elias el orden civilizatorio no depende de una conciencia funcional al proceso, como tampoco de un inconsciente que constriñe la interpretación de la realidad social, depende de las redes de interrelaciones e interdependencia que modelan la configuración del cambio social. Lo psíquico y lo social son dos aspectos de dicha transformación. Un punto de vista analítico similar sobre la interpretación de la historia nos ofrece Polanyi al analizar el mito liberal de una conspiración desarrollada por terratenientes, fabricantes y sindicalistas que habrían hecho sucumbir al libre mercado, que en la lógica de los liberales es un mecanismo natural y espontaneo mientras 
que la tendencia colectivista sería un efecto concertado. Polanyi plantea lo contrario al proponerse esclarecer que no existió ninguna conspiración por parte de intereses clasista para acabar con el libre mercado. Con lo cual critica la filosofía política de Marx y el interés clasista como eje del cambio social al igual que la filosofía política liberal que sostenía que el proteccionismo era el resultado de una acción seccional o concertada (Polanyi,2003).

Ambos obstruían una visión global de la sociedad de mercado y de la función del proteccionismo en tal sociedad. Para Polanyi son más importantes las necesidades de la "sociedad" a nivel global que la acción concertada de un sector de esta. En definitiva, si existen las condiciones estructurales de necesidad social pueden resultar las demandas de corte seccional e impulsar el cambio social, de otra forma este no se podría producir. Así, pues, los meros intereses clasistas para Polanyi no pueden ofrecer una explicación satisfactoria de ningún proceso social a largo plazo. Para Polanyi los intereses de clase se refieren principalmente a factores de necesidad social como calidad de vida, seguridad, posición o estatus, precisamente por ello, sectores de la "sociedad" se oponían al mercado autorregulado, porque este amenazaba los intereses sociales y no materiales. Este proteccionismo frente al libre mercado se producía por el daño letal causado a las instituciones donde está incorporada la existencia de los individuos. La causa de la degradación no es la explotación económica, como suele suponerse, sino la degradación del ambiente cultural de las víctimas. Es decir, el proteccionismo es una reacción contra una catástrofe social que es la instalación premeditada del libre mercado como mecanismo de regulación de la "sociedad". Un mecanismo de autoconservación que surge de forma natural por las condiciones de degradación de su medio social y cultural, producido por el mecanismo antinatural del mercado (Polanyi, 2003).

En esta misma lógica de cazadores indiciarios que pusieron el acento en lo antes no visto, relevo el punto de vista de Gabriel Tarde. Es un autor revalorizado contemporáneamente por autores como Deleuze, Latour y Lazaratto. Tonkonof (2011) señala que existiría una convergencia entre la microfísica del poder foucaultiano y la perspectiva de una microsociología de Tarde, aunque Foucault no lo haya referenciado. Esta convergencia se debe al uso del modo disciplinario del ejercicio del poder en Foucault y una microsociología propuesta por Tarde, preocupada por una multiplicidad de procesos, con frecuencia menores de origen diferente, de localización diseminada, que coinciden se repiten o se imitan, se apoyan unos sobre otros, se distinguen según su dominio de aplicación, entran en convergencia y dibujan poco a poco el diseño de un método general. En este sentido las disciplinas serian una anatomía política del 
detalle, hallazgos tecnológicos diversos que, combinándose, entrando en redes flexibles, difundiéndose a lo largo de la multiplicidad del cuerpo social, llegan a formar una sociedad disciplinaria. Para Tarde todo incluyendo los individuos es una sociedad.

La propuesta de la microsociologia de Tarde (2011) rechaza el recurso explicativo a factores impersonales o a las ideas de los grandes hombres, remplazándolo por las pequeñas ideas de los pequeños hombres, las pequeñas invenciones y las corrientes imitativas. Uno de los objetivos de esta perspectiva sociológica es criticar la idea de sociedad como un ente abstracto exterior a las dinámicas del acontecimiento, de la imitación, la invención y la oposición. Por ello es que remplaza la noción de sociedad por la de asociación o ensamblajes (Tonkonoff, 2011). La perspectiva de Tarde cuestiona la división entre cultura y naturaleza en relación a la comprensión de la interacción humana, también la distinción entre lo micro y lo macro para responder a la pregunta de cómo la sociedad es generada. Autores como Lazaretto consideran a la Tarde como un creador de una ontología del acontecimiento y de la multiplicidad, imprescindibles para la producción de nuevas formas de pensamiento sobre la "sociedad".

Como hemos podido ver en todos estos autores cazadores de lo indiciario de lo abierto, del estar siendo (indicios) existen ciertos paralelismos que es necesario explorar. Pero a modo tentativo podríamos señalar que en cada uno de ellos existe un enfoque que ve los fenómenos en la lógica del cazador indiciario que da cuenta de los detalles infinitesimales, los mechones de pelo, las huellas en el fango, es decir, los indicios, los detalles o anomalías pasadas por alto por la teoría convencional económica o social. Existe en estos autores una pretensión de rastrear lo abierto, lo que está en continua actuación y recreación, en las operatorias, los procedimientos, los modos de hacer o artes de hacer, los modos de empleo. El punto de vista de estos cazadores de lo indiciario, pensadores de los bordes de las disciplinas, establece la posibilidad "apreciar los entres o articulaciones" que acercan o entrelazan lo que anteriormente tenia delimitaciones rígidas. Permite pensar la transdisciplinariedad y conectar a esta con principios muchos más amplios, desde los cuales poder pensar una política emancipatoria, esbozando una serie de conceptos como posibilidad de interrogación, que conecta con las practicas "económico-sociales" de tipo libertario, solidario o democratizador, buscando ligar de otras formas la relación de la economía con la socialidad.

Este es un punto a seguir explorando...... 
Referencias bibliográficas

Certeau Michel (2000), La invención de lo cotidiano I. Artes de hacer, México, Universidad. Iberoamericana.

Elias Norbert (1994), El proceso de la civilización. Investigaciones sociogenéticas y psicogenéticas. México, Fondo de Cultura Económica.

Gaiger Inacio (2017), "El sentido metautilitarista de la Economía Social y Solidaria", en Raúl González (editor) Ensayos sobre economía cooperativa, solidaria y autogestionaria" (hacia una economía plural). (en edición).

González Raúl (2016), "La perspectiva y el debate sobre una economía cooperativa, solidaria y autogestionaria como centro de la economía social”, Santiago, Revista de la Academia No 21.

Godelier Maurice (1976), Antropología y economía, Editorial Anagrama, Barcelona.

Latour Bruno (2008), Reensamblar lo Social. Una introducción de la teoría del actor-red. Buenos Aires, Manantial.

Polanyi Karl (2003), La Gran transformación, Ciudad de México. Fondo de Cultura Económica,

Polanyi K., Alier J., M., Pearson H. W., Arensberger C. M., \& NICOLÁS A. (1976), Comercio y mercado en los imperios antiguos, Barcelona, Editorial Labor. E.P.

Tarde Gabriel (2011). Creencias, deseos, sociedades. Buenos Aires, Cactus.

Santiago Silviano (2012), "Interpretando interpretaciones de América Latina", en Una literatura de los Trópicos. Ensayos de Silviano Santiago, Santiago de Chile, Ediciones Escaparate. 